\title{
Prediction of maximum wave-induced liquefaction in porous seabed using Multi-Artificial Neural Network model
}

\author{
Daeho (Fred) Cha, Hong Zhang † and Michael Blumenstein ${ }^{\ddagger}$
}

July 5, 2010

\section{Abstract}

In the last few decades, considerable efforts have been devoted to the phenomenon of wave-induced liquefactions, because it is one of the most important factors for analysing the seabed and designing marine structures. Although numerous studies of wave-induced liquefaction have been carried out, comparatively little is known about the impact of liquefaction on marine structures. Furthermore, most previous researches have focused on complicated mathematical theories and some laboratory work. In the present study, a data dependent approach for the prediction of the wave-induced liquefaction depth in a porous seabed is proposed, based on a multi-artificial neural network (MANN) method. Numerical results indicate that the MANN model can provide an accurate prediction of the wave-induced maximum liquefaction depth with $10 \%$ of the original database. This study demonstrates the capacity of the proposed MANN model and provides coastal engineers with another effective tool to analyse the stability of the marine sediment.

\footnotetext{
${ }^{*}$ Griffith School of Engineering, Griffith University Gold Coast Campus, Australia

†Corresponding author: hong.zhang@griffith.edu.au, Tel:+61(07)55529015, Fax:+61(07)55528065, Griffith School of Engineering, Gold Coast Campus, Griffith University, QLD 4222, Australia

${ }^{\ddagger}$ School of Information and Communication Technology, Griffith University Gold Coast Campus, Aus-
} tralia 
KEYWORDS: Wave-induced liquefaction, Artificial neural networks, Multi-artificial neural network.

\section{Introduction}

Most marine structures such as breakwaters and seawalls, have been used for the protection of coastal communities against natural disasters like flooding or erosion. Design of coastal structures against the wave loading has been intensively studied and improved in the past. However, the damage of such structures still occurs due to the failure of foundation around the structures. Wave-induced seabed liquefaction, which has been recognised as the culprit of foundation failure, has been of profound interest to coastal and geotechnical engineers recently (Jeng 2003).

Since the 1970's, numerous investigations for wave-induced liquefaction have been carried out. Bjerrum (1973) was possibly the first person who recognised and analysed wave-induced liquefaction occurring in saturated seabed sediments in connection with foundation design in the North sea. Later, Ishiara and Yamazaki (1984) suggested a methodology of evaluating the magnitude of cyclic stress and wave-induced liquefaction on the basis of storm parameters, which is based on the theoretical solution of Yamamoto et al. (1978). Zen et al. (1985) proposed that wave-induced liquefaction of foundations, with its subsequent slip circle failure, was one of the possible causes of the collapse of structures. Their results indicated that liquefaction might be induced by ocean waves only under the condition that the pore pressure dissipation or redistribution was restricted by the existence of layers with low permeability. Later, Taotsos et al. (1989) evaluated pore pressure generation and liquefaction potential in the seabed floor due to cyclic wave action using a numerical model. Their results concluded that the soil permeability had a very significant influence on pore-water pressure generation and liquefaction because high 
permeability prevented the development of excess pore-water pressure. Zen and Yamazaki (1993), using a finite difference method, investigated the mechanism of the wave-induced liquefaction and densification in a permeable seabed. From the results presented, they concluded that the liquefaction and densification occurred alternately in the seabed even in one period of the wave loading. The authors (Jeng and Zhang 2005, Zhang and Jeng 2005) established an integrated three-dimensional model, incorporating a wave model and soil model, to investigate the wave-induced liquefaction potential in the Gold Coast region in Australia. Both non-breaking and breaking waves were considered in their model. Also, Liu and Jeng (2007) using a simple semi-analytical model for the random waveinduced soil response in unsaturated seabed of finite thickness. Most recently, Noorzad et al. (2009) analysed a wave-induced liquefaction carried out using mechanisms similar to earthquake-induced liquefaction.

Recently, artificial neural networks (ANNs) have found extensive utilisation in solving many complex real-world engineering problems, such as the prediction of rainfall intensity (French et al. 1992), the prediction of the environmental properties of the output stream from a wastewater treatment plant (Gontarski et al. 2000), tide-forecasting (Lee and Jeng 2002, Lee et al. 2002, Joorabchi et al. 2007), the prediction of the settlement of shallow foundations on cohesionless soils (Mohamed et al. 2002), and predictions of typhoon storm surge using ANNs (Lee 2006).

For the liquefaction consideration, ANN models have been applied to the prediction of seismic liquefaction potential by using a back-propagation neural network (Goh 1995). Juang and Chen (1999) also predicted seismic liquefaction potential from cone penetration field test data. Cha et al. (2004) and Jeng et al. (2004) were possibly the first to apply ANNs for the prediction of wave-induced liquefaction in a porous seabed. Their research demonstrated the capability of ANN models for the prediction of the maximum waveinduced liquefaction depth within various wave and soil conditions. The authors (Cha 
et al. 2006, Cha etal 2006, Zhang et al. 2007. Cha et al. 2008) established prediction of wave-induced liquefaction depth using various ANN techniques. However, the SANN model is less accurate for small and large liquefaction depths.

The goal of this study is to develop a multi-artificial neural network model to predict the wave-induced liquefaction potential, especially the maximum liquefaction depth using the results obtained from the existing data from the author's previous study (Cha 2003). Subsequently, a MANN model will be investigated for the improvement of the accuracy over the SANN model. Finally, a series of numerical experiments has been conducted to demonstrate the capability of the proposed MANN model.

\section{Wave-induced soil response}

In this study, we consider an ocean wave propagating over a porous seabed of finite thickness. The definition of the problem is illustrated in Figure 1. The porous seabed is treated as hydraulically isotropic with the same permeability in all directions. Zienkiewicz et al. (1980) presented a general set of governing equations, which describe the behaviour of a linear elastic porous solid under dynamic conditions. These equations are summarised in a tensor form as below

$$
\begin{aligned}
& \sigma_{i j, j}=\rho \ddot{u}_{i}+\rho_{f} \ddot{w}_{i}, \\
& -p, i=\rho_{f} \ddot{u}_{i}+\frac{\rho_{f}}{n} \ddot{w}_{i}+\frac{\rho_{f} g}{k_{z}} \dot{w}_{i}, \\
& \dot{\varepsilon}_{i i}+\dot{w}_{i, i}=-\frac{n}{K_{f}} \dot{p},
\end{aligned}
$$

where $p$ is the pore pressure in excess of hydrostatic, $n$ is the porosity, $\rho$ is the combined soil density; $\rho_{f}$ is the fluid density, $u$ and $w$ are the displacements of solid and relative 
displacements of solid and pore fluid, $\varepsilon_{i i}$ is the element of soil strain tensor, and $k_{z}$ is the soil permeability. $1 / K_{f}$ is the compressibility of pore-fluid, which is defined by

$$
\frac{1}{K_{f}}=\frac{1}{2 \times 10^{9}}+\frac{1-S}{P_{w o}}
$$

in which $S$ is the degree of saturation, $P_{w o}$ is the absolute water pressure. The definition of effective stresses, $\sigma_{i j}^{\prime}$, which are assumed to control the deformation of the soil skeleton are given by the total stress $\left(\sigma_{i j}\right)$ and pore pressure $(p)$ as,

$$
\sigma_{i j}=\sigma_{i j}^{\prime}-\delta_{i j} p
$$

where $\delta_{i j}$ is the Delta denotation. It is noted that the tensile stresses are represented as positive signs, while pore pressure is compression positive in this study.

Therefore, the equation of force balance, equation (1) becomes

$$
\sigma_{i j, j}^{\prime}=\delta_{i j} p_{, i}+\rho \ddot{u}_{i}+\rho_{f} \ddot{w}_{i}
$$

To obtain the wave-induced pore pressure, soil and fluid displacements involved in (1)-(3), appropriate boundary conditions are required. The boundary conditions are summarised below,

$$
\begin{aligned}
& u=w=0, p=0 \text { as } z \rightarrow-\infty \\
& p=P_{b}, \sigma_{z}^{\prime}=\tau=0 \text { at } z=0
\end{aligned}
$$

where, $P_{b}$ is the wave pressure at the seabed surface, which is given by the equation below:

$$
P_{b}=\frac{\gamma_{w} H}{\cosh k d} \cos (k x-\omega t)
$$

where $H$ is the wave height and $d$ is the water depth, $k$ is the wave number, $\omega$ is wave frequency, and $\gamma_{w}$ is the unit weight of water. 
Following the procedure in Cha (2003), the general solution for the soil and pore fluid displacements, to satisfy the bottom boundary condition (7), can be expressed as

$$
\begin{aligned}
& \bar{U}_{x}=a_{1} e^{\lambda_{1} \bar{z}}+a_{3} e^{\lambda_{2} \bar{z}}+a_{5} e^{\lambda_{3} \bar{z}} \\
& \bar{U}_{z}=a_{1} b_{1} e^{\lambda_{1} \bar{z}}+a_{3} b_{3} e^{\lambda_{2} \bar{z}}+a_{5} b_{5} e^{\lambda_{3} \bar{z}} \\
& \bar{W}_{x}=a_{1} c_{1} e^{\lambda_{1} \bar{z}}+a_{3} c_{3} e^{\lambda_{2} \bar{z}}+a_{5} c_{5} e^{\lambda_{3} \bar{z}} \\
& \bar{W}_{z}=a_{1} d_{1} e^{\lambda_{1} \bar{z}}+a_{3} d_{3} e^{\lambda_{2} \bar{z}}+a_{5} d_{5} e^{\lambda_{3} \bar{z}}
\end{aligned}
$$

where $\lambda_{i}$ coefficients are the roots of the characteristics equation from the couple of equation. Based on the wave-induced soil and fluid displacements, we can obtain the waveinduced pore pressure, effective stresses and shear stress. The unknown coefficients, $a_{i}$, $b_{i}, c_{i}$ and $d_{i}$, can be solved with the boundary condition. Once we obtain coefficients, we can calculate the wave-induced soil response parameters. Detailed information of the above solution can be found in Jeng and Cha (2003).

\subsection{Wave-induced liquefaction}

It has generally been accepted that, when the vertical effective stress vanishes, the soil will be liquefied. Under such a situation, the soil matrix loses its strength to carry any load and consequently causes seabed instability. However, the mechanisms of the wave-induced soil liquefaction in marine sediments have not been clearly addressed in geotechnical terms at the present stage. The liquefaction is also affected by the state of soil compaction, permeability, the wave-induced cyclic stress as well as the degree of drainage.

To apply the concept of excess pore pressure to a seabed, a schematic drawing of the pore pressure and effective stress distributions is illustrated in Figure 2. The solid curves in the figure indicate the pore pressure beneath a wave trough and a wave crest. The excess pore pressure is transient in nature, because both $P_{b}$ and $p$ are oscillatory and 
periodical in real ocean environments. Consequently, the effective stress varies periodically in accordance with the change of the excess pore pressure. If it attains zero or a negative value at certain depths below the seabed surface, the soil skeleton will reach a liquefied state (Figure 2). Thus, the liquefaction criterion can be expressed as (Jeng 1997, Zen 1990)

$$
-\frac{1}{3}\left(\gamma_{s}-\gamma_{w}\right)\left(1+2 K_{o}\right) z+\left(P_{b}-p\right) \leq 0 .
$$

where $K_{o}$ is the coefficient of earth pressure at rest, which is normally varied from 0.4 to 1.0 and 0.5 is commonly used for marine sediments (Scott 1963).

Although other criteria of the wave-induced liquefaction have been proposed based on the concept of effective stresses (Okusa 1995, Tsai 1995), the criterion based on the concept of excess pore pressure (Zen and Yamazaki 1990, Jeng 1997) was demonstrated to be a better criterion of liquefaction, comparing with the field data (Jeng 1997).

\section{Artificial neural networks}

Artificial neural networks (ANNs) are computing systems made up of a number of simple, highly interconnected processing elements, which process information by their dynamic state response to external inputs (Caudill 1987). ANNs have been developed since the early 1940's, they were not applied to real-world problems until the middle of the 1980's when algorithms became sophisticated enough for general applications.

Figure 3 illustrates an artificial neuron in comparison with a basic human brain neuron. It is clearly shown that artificial neurons are based on the most simplest functions of the human brain. An ANN model can be built, based on the artificial neuron (Fig 3(b)). Figure ?? shows that a typical ANN model includes an input layer, hidden layer(s) and output layer. Each layer is made up of several neurons and the layers are interconnected by 
sets of corresponding weights. The input layer neurons receive initial input information, after which, outputs may be obtained by using various transfer functions. In this paper, we adopted the logarithmic sigmoid transfer function is adopted, which can be expressed as

$$
f(x)=\frac{1}{1+e^{-x}}
$$

In the learning procedure, which is also called training, using an appropriate data set, the interconnection weights are adjusted based on the interaction between input and output values, which is an important part of the ANN model. In this study, the backpropagation algorithm is applied. The back-propagation network is the most representative model for various applications of artificial neural networks. The back-propagation algorithm can be applied to networks that contain at least one hidden layer, and fully connected units in each layer. The main procedure of the back-propagation network is that the error at the output layer continuously propagates backward to the input layer through the hidden layer(s) in the network to obtain the final desired response. It clearly means that the goal of this procedure is to obtain a desired output when certain inputs are given. The error function, which is used in this paper, is given as

$$
E=\frac{1}{M} \sum_{i=1}^{M}\left(D_{i}-O_{i}\right)^{2}
$$

where $D_{i}$ and $O_{i}$ are the desired and the actual output values respectively, $M$ is the total number of training data set. Since the error is the difference between the actual output and the target output, the error produced depends on the configuration of the weights, and hence it is necessary to adjust the weights in order to minimize the error. The method of gradient descent is employed to adjust the weights. The details of the back-propagation algorithm can be found in Rumelhart et al. (1986). 


\subsection{ANN model for wave-induced liquefaction}

Wave-induced seabed liquefaction was previously predicted by solving complicated mathematical equations. However, the existing deterministic models have been based on various assumptions, which have limited the application of the models. The ANN model does not involve all the physical equations, that only requires reliable input data, which are obtained from previous historic, field data or numerical solutions. In this study, a database was generated from the numerical model described in section 2. The structure of the proposed ANN model, which is used in this study, is illustrated in Figure 4. A multi-layered back-propagation network with sigmoidal activation functions is used, which is the most popular scheme employed in engineering prediction or forecasting problems.

The parameters used as inputs to the ANN model are permeability (fixed value), the degree of saturation, the seabed depth, the wave period, the wave height, and the water depth. It has been reported that soil permeability is very sensitive to the occurrence of the wave-induced liquefaction potential (Jeng 1997). In general, permeabilities of different soils are in different order, which make the procedure of normalisation to be difficult. After preliminary tests of ANN models, we found that inclusion of permeability cause the difficulties of convergence of the model. Thus, we have an ANN model for each case with a fixed value of soil permeability. This will make the proposed ANN model much easier to converge.

In this study, a multi-artificial neural network (MANN) is introduced, which is the extention of the Single-artificial neural network (SANN) developed in the author's previous study (Jeng and Cha 2003a). The major difference between the SANN model and the MANN model is the number of networks used. The SANN model is comprised of only one network which is used for the whole database, and the MANN is composed of various networks depending on the range of the database. 


\subsection{Numerical simulations}

The performance of ANN models depends on the number of hidden layers, the learning factors, the number of training iterations (epochs), the weight configurations and the number of neurons in each layer. These factors, for this study, are tabulated in Table 1. The ANN model is established using the MATLAB ANN ToolBox environment. The ANN is a data driven model which has to be trained or verified for each site. If there is not enough field data, the predicted information can only be used as a reference

The input data for the poro-elastic model, which establishes the database of the ANN model, are shown in Table 2. As seen in the table, the data set has covered most of the possible ranges of wave and soil conditions. Three different soil permeabilities, representing three different sandy seabed (coarse and fine sand), are used in this study. The poro-elastic numerical model test results indicate that wave-induced liquefaction occurs in numerous cases. The whole database contains approximately 50,000 cases of wave-induced maximum liquefaction depth.

In this case study, we chose a data set of size 20,000 (approximately) of maximum liquefaction depth from the poro-elastic numerical model. From this database, $80 \%$ of the data is used for the training procedure, and the remaining data is for model verification.

\subsubsection{Data acquisition}

In this study, a database including wave, soil parameters and maximum liquefaction depth is generated from a numerical model based on various parameters (Table 2). However, the ANN model had a limitation of input and output values between 0 and 1 or -1 and 1 , due

to the ANN's activation functions, which is a main aspect of the model. Therefore, the input and output values are converted to suit this range. For example, $60 \mathrm{~m}$ for seabed thickness is converted to 0.6 hundred meters. It is a clearly shown that the ANNs required 
appropriate data acquisition and preprocessing steps to build a database for training, even if good quality data was collected.

\subsubsection{Single-artificial neural network model (SANN)}

The advantages of the single-network model (Cha et al. 2004), were its simplicity and limited time consumption. The test results for prediction of the maximum liquefaction depth and the training procedure using the proposed SANN model are presented in Figures $5-7$.

Figure 5(a) illustrates the convergence of the training procedure. It is clearly shown that the training error is less than $10^{-3}$, which is based on Eq.(16). It implies that the SANN weight configuration can be used to forecast the maximum liquefaction depth with a good accuracy. Figure 5(b) represents the prediction of the maximum liquefaction depth $\left(Z_{L}\right)$ using the ANN model versus the poro-elastic numerical maximum liquefaction

depth $\left(Z_{L}\right)$. As seen in this figure, the prediction of maximum liquefaction agrees with the numerical calculation data overall.

Although the statistical analysis suggests a good correlation between the SANN simulation results and the original database from the poro-elastic model, it can be seen from the Figures (Fig(5)-Fig(7)) that there is a significant disagreement between the SANN modelling results and the original database at the extremities (i.e., small and large liquefaction depths).

\subsubsection{Multi-artificial neural network model (MANN)}

The occurrence of wave-induced liquefaction depends on soil and wave parameters. However, as shown in Figures (5)-(7), SANN model fails to predict values near the zero liquefaction and over $3 \mathrm{~m}$ liquefaction depth range. Therefore, MANN model is applied 
to deal with three different ranges, from 0 to $1 m, 1$ to $3 m$ and 3 to $5 m$, with three different networks. Figure 8 illustrates the construction of the MANN model. These ranges are based on preliminary tests, and finalise it to be the best option for this problem. It has a similar training procedure to the SANN model, but the data used for training is divided into 3 different training databases (Figure 9).

To apply the MANN, the ANN model for the range of 1-3 $m$ will be used for the testing database first, because most cases locate in the range. If the results obtained from the model of 1-3 $m$ is out of the range, we can use another two models to further confirm the results with new range of maximum liquefaction depth.

Figure 10 clearly shows that each range of maximum liquefaction depth of the MANN predicted results agrees with the numerical calculation depths. It is obvious that maximum liquefaction depth ranges between 0 to $1 m$ and 3 to $5 m$ have a good agreement between the MANN and poro-elastic models. It also shows that the correlation of the MANN model and the poro-elastic model is over $97 \%$ for all ranges. These figures illustrate that difference of the predictions of maximum depths are within the $\pm 10 \%$ range, which is acceptable for an engineering application. The results indicate that if we can control the range of data in each case, the accuracy for the ANN model can be improved. In other words, if the weights for each case can be optimised, the accuracy of prediction will be dramatically increased. Similar trends for other ranges of $K_{z}$ have been observed in Figures 10-11.

The correlation between the SANN model simulation results and original analytical solutions is similar to that obtained between the MANN model simulation results and the original database. Hence, a RMSE (Root Mean Square Error) is introduced to distinguish the difference between SANN and MANN model. The RMSE is defined as

$$
R M S E=\sqrt{\frac{1}{N} \sum_{i=1}^{N}\left(L_{A}-L_{P}\right)^{2}}
$$


where $L_{A}$ and $L_{P}$ are the liquefaction depths from the ANN model and the poro-elastic model respectively; $N$ is the total number of liquefaction depth data. It is clearly shown that the RMSE values of the SANN model $(0.17 m$ and $0.19 m)$ are twice as large as those of the MANN model results $(0.085 \mathrm{~m}$ and $0.076 \mathrm{~m})$ as shown in Figures (Fig(10)-Fig(11)). The SANN model encompasses the whole range of maximum liquefaction depth (0 to $5 \mathrm{~m}$ ). This clearly shows that when predicting the liquefaction depth at the extremes (less than $1 m$ or greater than $3 m$ ), the SANN model decreases in accuracy. Hence, this has necessitated the use of the MANN model for these situations.

\section{Conclusions}

In this paper, we established and compared SANN and MANN models, and applied these models to the prediction of the wave-induced liquefaction potential in a porous seabed. ANNs are still fresh and an un-explored area in engineering. The results in this pa-

per clearly show that the MANN model provides good accuracy in the prediction of the wave-induced maximum liquefaction depth. It also shows that ANNs can be a powerful engineering tool in future research. Unlike conventional engineering mechanics approaches, ANN models do not require a complicated mathematical procedure. Numerical examples have demonstrated that MANN model is applicable for the prediction of the wave-induced maximum liquefaction depth with reasonable accuracy.

\section{References}

Bjerrum J., 1973. Geotechnical problem involved in foundations of structures in the North Sea. Géotechnique. 23(3), 319-358

Caudill M., 1987. Neural Networks primer, Part I. AI expert. Miller Freeman, San Francisco. 
Cha D.H., 2003. Mechanism of Ocean Waves Propagating over a Porous Seabed. MPhill Thesis. Griffith University, Australia.

Cha D.H., Jeng D.S., Blumenstein M., 2004. Assessment of wave-induced liquefaction in a porous seabed: Application of an ANN model. Asian Journal of Information Technology. 3(5), 386-399.

Cha D.H, Blumenstein M., Zhang H., and Jeng D.S., 2006. Improvement of an Artificial Neural Network Model using Min-Max Preprocessing for the Prediction of Wave-induced Seabed Liquefaction. 2006 IEEE World Congress on Computational Intelligence and 2006 International Joint Conference on Neural Networks, Vancouver, Canada, 16-21.

Cha D.H., Jeng D.S., Blumenstein M., and Zhang H., 2006. Evaluation of wave-induced liquefaction in a porous seabed: Using an Artificial Neural Network and a Genetic Algorithm -based model. The Proceedings of The Sixteenth International OFFSHORE AND POLAR ENGINEERING CONFERENCE, San Francisco, California, USA, 302-308.

Cha D.H., Blumenstein M., Zhang H., Jeng D.S., 2008. A Neural-Genetic Technique for Coastal Engineering: Determining Wave-induced Seabed Liquefaction Depth, Book Chapter, Engineering Evolutionary Intelligent Systems, Springer, SCI Series, 337-351, 2008.

French N.M., Witol F., Krajewski F.W., Cuykendallb R.R., 1992. Rainfall forecasting in space and time using a neural network. Journal of Hydrology. 137, 1-31.

Goh A.T.C., 1995. Seismic liquefaction potential assessed by neural networks. Journal of Geotechnical Engineering, ASCE. 120, 1467-1480. 
Gontarski C.A., Rodrigues P.R., Mori M., Prenem L.F., 2000. Simulation of an industrial wastewater treatment plant using artificial neural networks. Computers and Chemical Engineering. 24, 1719-1723.

Jeng D.S., 1997. Wave-induced seabed instability in front of a breakwater. Ocean Engineering. 24(10), 887-917.

Jeng D.S., and Cha D.H., 2003a. Wave-induced seafloor dynamics. Applied Mechanics Review. 56(4), 407-429.

Jeng D.S., Cha D.H., 2003. Effects of dynamic soil behaviour and wave non-linearity on the wave-induced pore pressure and effective stresses in porous seabed. Ocean Engineering. 30(16), 2065-2089.

Jeng D.S., Cha D.H., Blumenstein M., 2004. Neural Network model for the prediction of the wave-induced liquefaction potential in a porous seabed. Ocean Engineering. 31(17-18), 2073-2086.

Jeng D.S., Zhang H., 2005. An integrated three-dimensional model for wave-induced pore pressure and effective stresses in a porous seabed. II: Breaking waves. Ocean Engineering. 32(16), 1950-1967.

Joorabchi A., Zhang. H. and Blumenstein M., 2007. Application of artificial neural networks in flow discharge prediction for the Fitzroy River, Australia, Journal of Coastal Research, SI 50, 287-291.

Juang C.H., Chen C.J., 1999. CPT-based liquefaction evaluation using neural network. Journal of Computer-Aided Civil Infrastructure Engineering. 14, 221-229. 
Lee T.L., 2006. Predictions of typhoon storm surge in Taiwan using artificial neural networks. Advances in Engineering Software. 40, 1200-1206.

Lee T.L., Jeng D.S., 2002. Application of artificial neural networks in tide-forecasting. Ocean Engineering. 29(9), 1003-1022.

Lee T.L., Tsai C.P., Jeng D.S., Shieh R.J., 2002. Neural network for the prediction and supplement of tidal record in Taichung Harbor, Taiwan. Advances in Engineering Software. 33, 329-338.

Liu H, and Jeng D.S., 2007. A semi-analytical solution for random wave-induced soil response and seabed liquefaction in marine sediments. Ocean Engineering, 34, 12111224 .

Mohamed S.K., Holger R.M., Mark B.J., 2002. Predicting Settlement of Shallow Foundations using Neural Networks. Journal of Geotechnical and Geo environmental Engineering. 128(9), 785-793.

Noorzad R., Safari S., and Omidvr M., 2009. The effect of structures on the waveinduced liquefaction potential of seabed sand deposits. Applied Ocean Reserch 31, 25-30.

Okusa S., 1998. Wave-induced stresses in unsaturated submarine sediments. Géotechnique. $35(4), 517-532$.

Rumelhart D.E., Hinton G.E., Williams R.J., 1986. Learning representations by backpropagating errors. Nature. 323, 53-536.

Scott R.F., 1963. Principle of Soil Mechanics. Addison-Publishing. Massachussetts. 
Taotsos S., Georgiadis M., Damakindow A., 1989. Numerical analysis of liquefaction potential of partially drained seafloor. Coastal Engineering. 13, 117-128.

Tsai C.P., 1995. Wave-induced liquefaction potential in a porous seabed in front of a breakwater. Ocean Engineering. 22, 1-18.

Yamamoto T., Koning H.L., Sellmejjer H., Hijum E.V., 1978. On the response of a poro-elastic bed to water waves. Journal of Fluid Mechanics. 87, 193-206.

Zen K., Yamazaki H., 1990. Mechanism of wave-induced liquefaction and densification in seabed. Soils and Foundations. 30, 90-104.

Zen K., Yamazaki H., 1993. Wave-induced liquefaction in a permeable seabed. Report, port and Harbour Research Institute. 31, 155-192.

Zhang H., Jeng D.S., 2005. An integrated three-dimensional model for wave-induced pore pressure and effective stresses in a porous seabed. I: A sloping seabed. Ocean Engineering. 32(5-6), 701-729.

Zhang H., Jeng D.-S., Cha D. and Blumenstein M., 2007. Parametric study on the Prediction of Wave-induced Liquefaction using an Artificial Neural Network Model 1, SI 50, Journal of Coastal Research, 374-378.

Zienkiewicz O.C., Chang C.T., Bettess P., 1980. Drained, undrained, consolidating and dynamic behaviour assumption in soils. Géotechnique. 30(4), 385-395. 


\begin{tabular}{ll} 
Table 1: Structure of ANN model \\
\hline \multicolumn{2}{c}{ Training model } \\
\hline Number of input neurons & 5 \\
Number of output neurons & 1 \\
Number of hidden units & 4 \\
Number of hidden layer & 5 \\
Learning rate & 0.5 \\
Momentum factor & 0.2 \\
Epochs & 9000 \\
\hline
\end{tabular}

Table 2: Input data of theoretical model

\begin{tabular}{|c|c|}
\hline \multicolumn{2}{|c|}{ Wave characteristics } \\
\hline Wave period $(T)$ & Varying between $5 \mathrm{sec}$ and $15 \mathrm{sec}$ \\
\hline Wave height $(H)$ & Varying between $0.5 m$ and $8 m$ \\
\hline Water depth $(d)$ & Varying between $10 \mathrm{~m}$ and $50 \mathrm{~m}$ \\
\hline \multicolumn{2}{|c|}{ Soil characteristics } \\
\hline Soil permeability $\left(K_{z}\right)$ & $10^{-4}, 5 \times 10^{-4}, 5 \times 10^{-6} \mathrm{~m} / \mathrm{sec}$ \\
\hline Seabed thickness $(h)$ & Varying between $10 \mathrm{~m}$ to $60 \mathrm{~m}$ \\
\hline Shear modulus $(G)$ & $10^{7} \mathrm{~N} / \mathrm{m}^{2}$ \\
\hline Poisson's ratio $(\mu)$ & 0.4 \\
\hline Porosity $(n)$ & 0.4 \\
\hline Degree of saturation $(S)$ & Varying between 0.95 and 1.0 \\
\hline
\end{tabular}




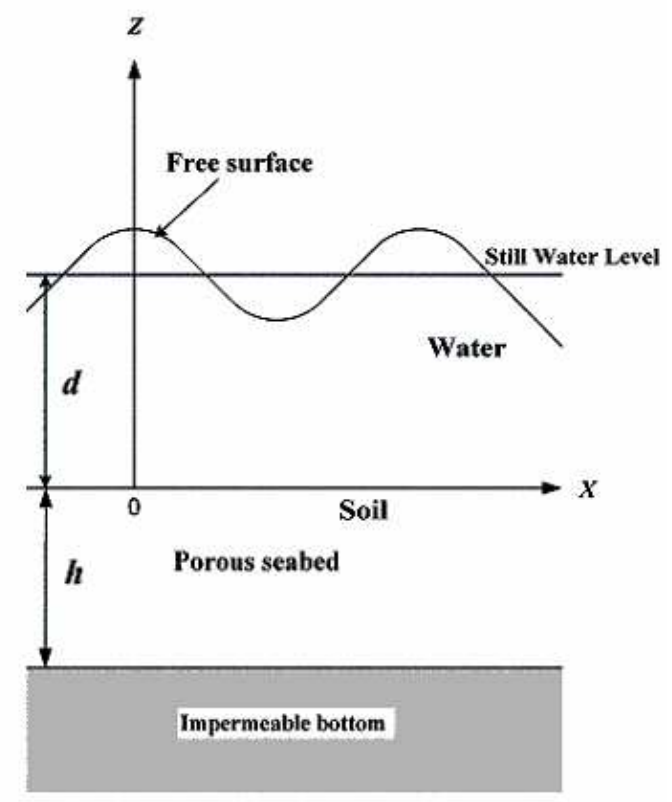

Figure 1: Definition of wave-seabed interaction.

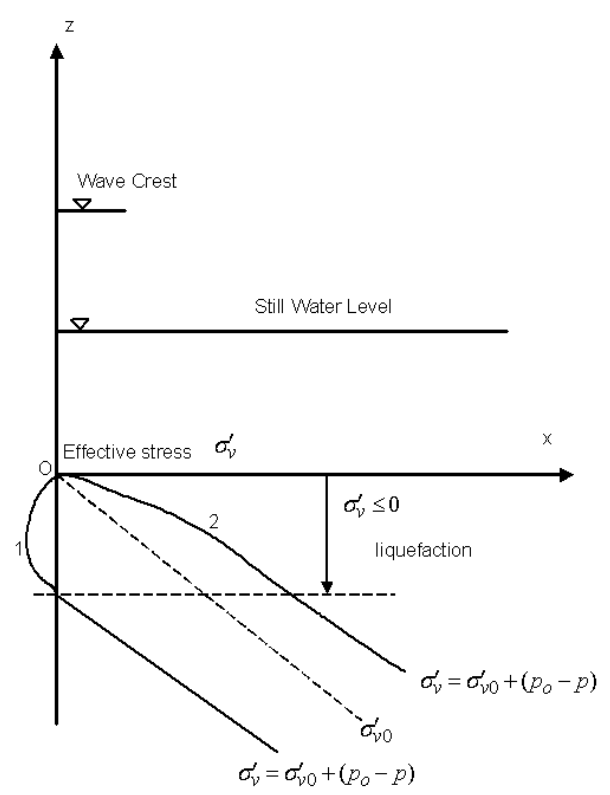

(a) Concept of excess pore pressure

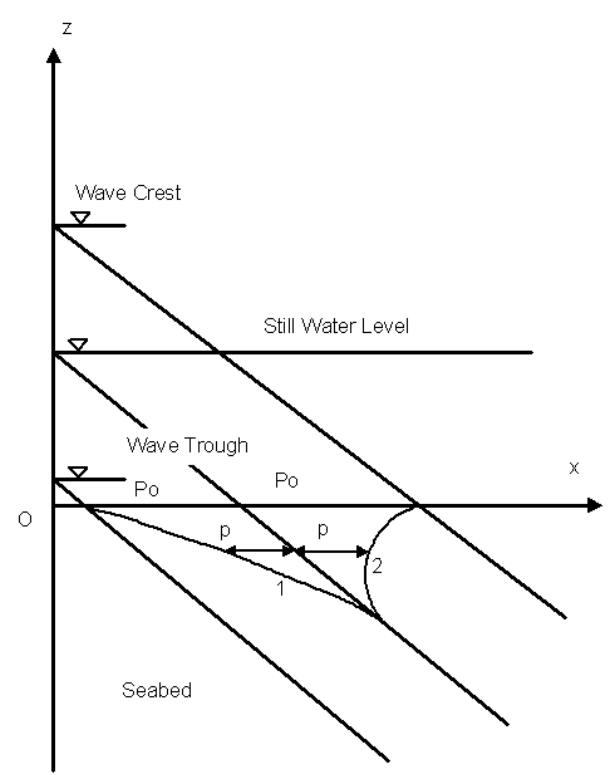

(b) Criterion of liquefaction

Figure 2: The concept of excess pore pressure and criterion of liquefaction(After Zen and Yamazaki (1990)) 


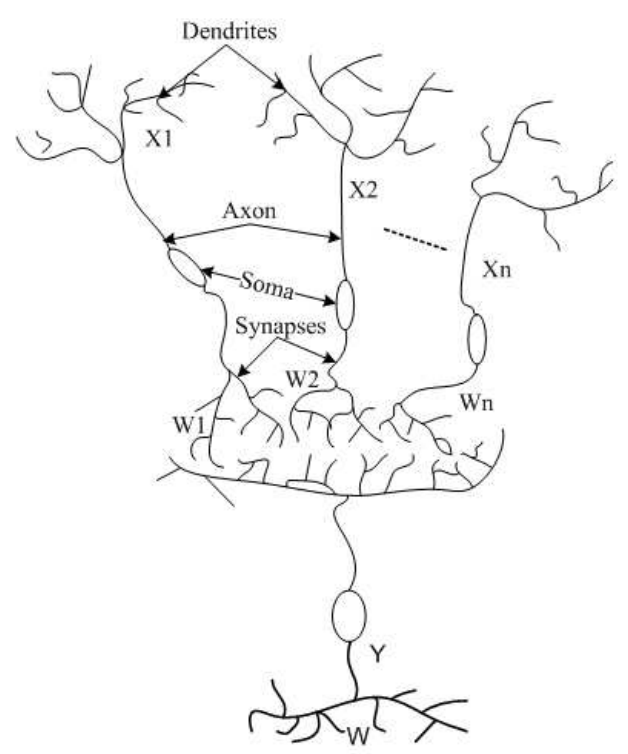

(a)

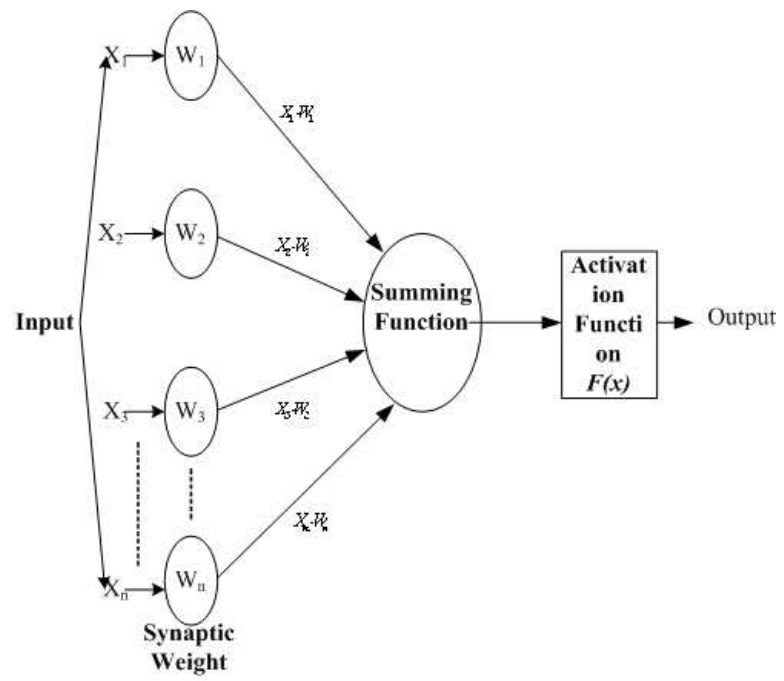

(b)

Figure 3: (a) Biological neuron from human brain. (b)Basic artificial neuron.

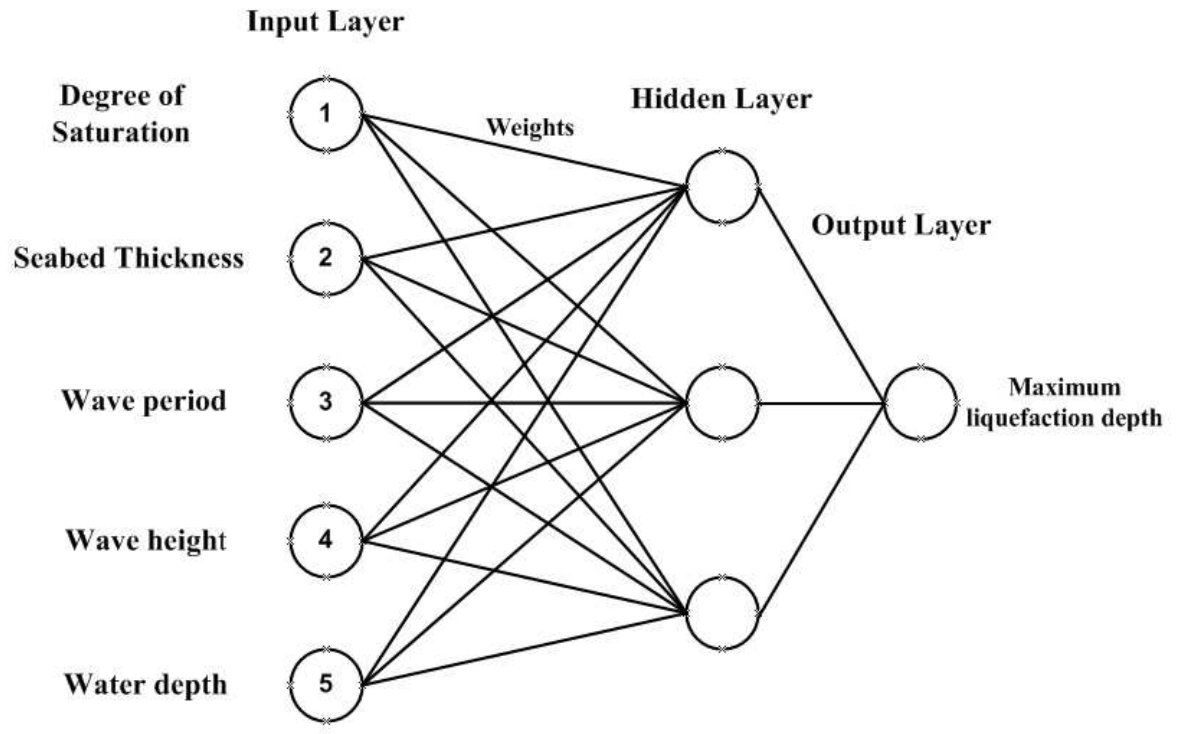

Figure 4: Architecture of the proposed ANN model for wave-induced liquefaction. 


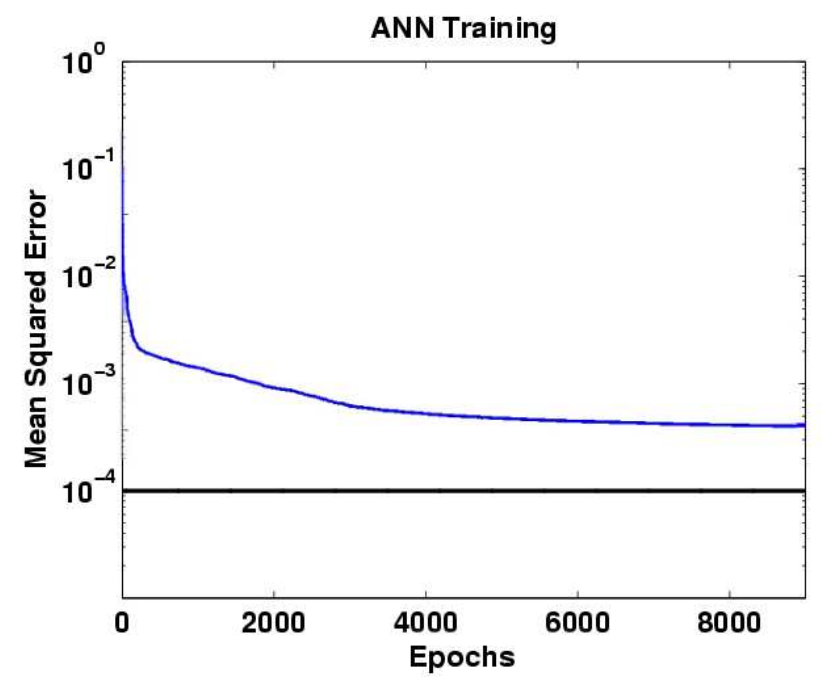

(a) Convergence of training data

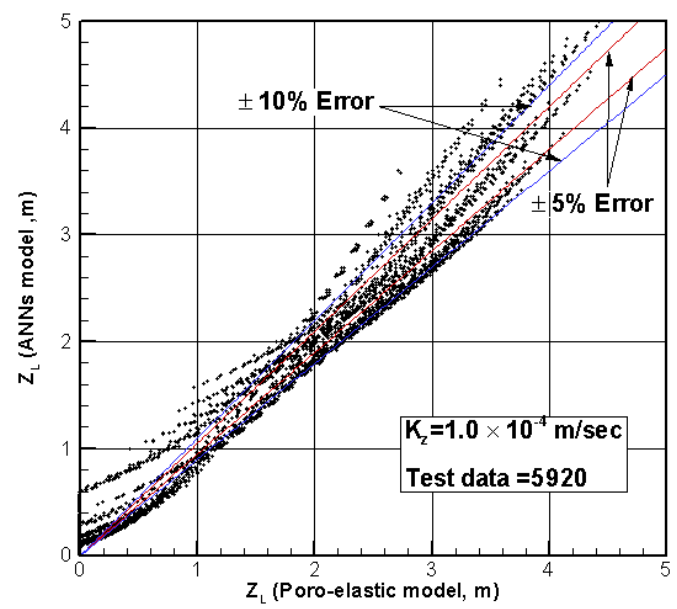

(b) Prediction of the SANN model versus the poro-elastic model

Figure 5: (a) Convergence of training data and (b) comparison of the wave-induced maximum liquefaction depth by the SANN model versus the poro-elastic model $\left(K_{z}=\right.$ $\left.10^{-4} \mathrm{~m} / \mathrm{sec}\right)$. 


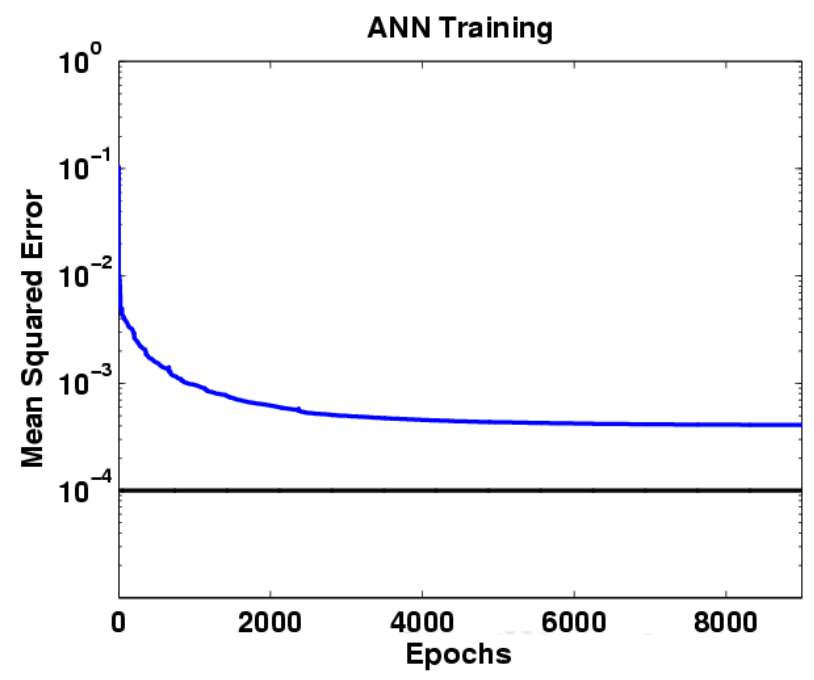

(a) Convergence of training data

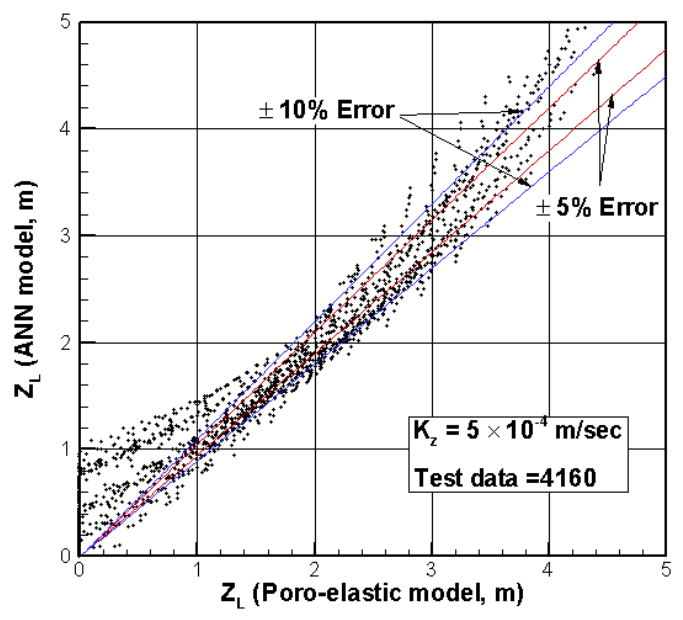

(b) Prediction of the SANN model versus the poro-elastic model

Figure 6: (a) Convergence of training data and (b) comparison of the wave-induced maximum liquefaction depth by the SANN model versus the poro-elastic model $\left(K_{z}=\right.$ $\left.5 \times 10^{-4} \mathrm{~m} / \mathrm{sec}\right)$. 


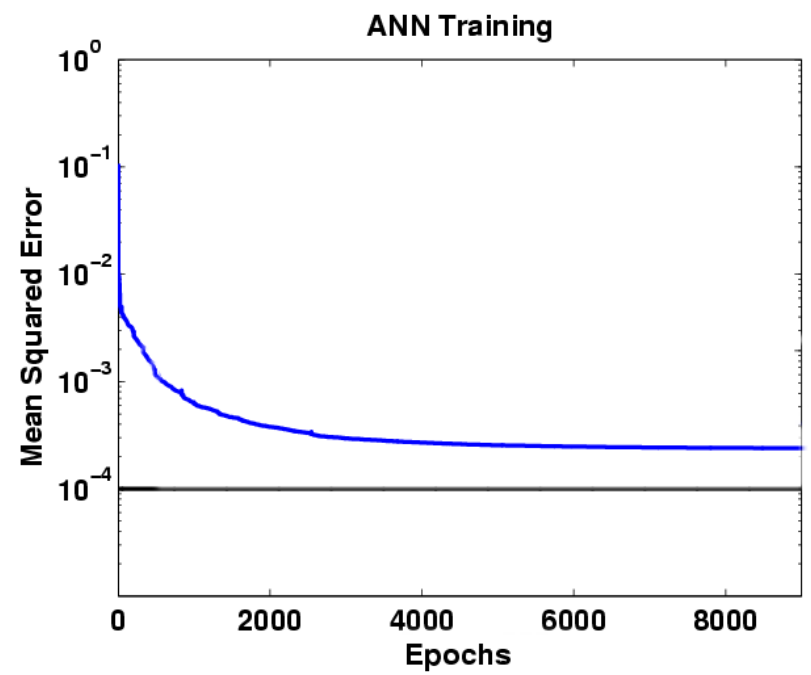

(a) Convergence of training data

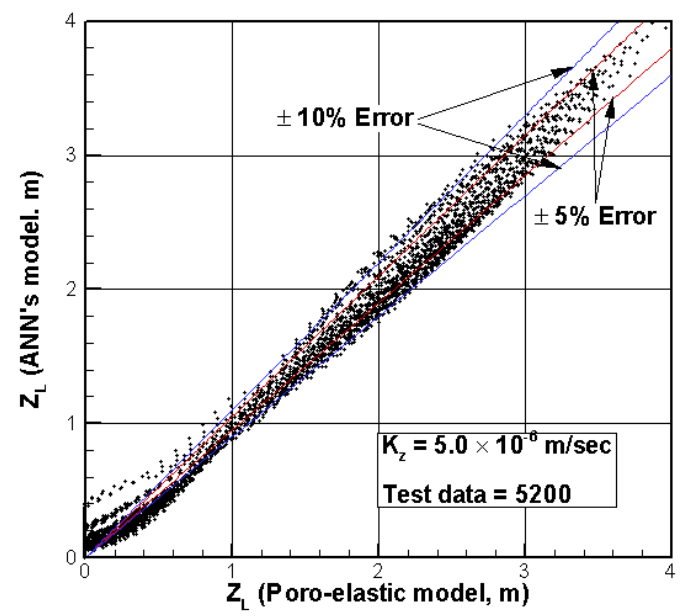

(b) Prediction of the SANN model versus the poro-elastic model

Figure 7: (a) Convergence of training data and (b) comparison of the wave-induced maximum liquefaction depth by the SANN model versus the poro-elastic model $\left(K_{z}=\right.$ $\left.5 \times 10^{-6} \mathrm{~m} / \mathrm{sec}\right)$. 


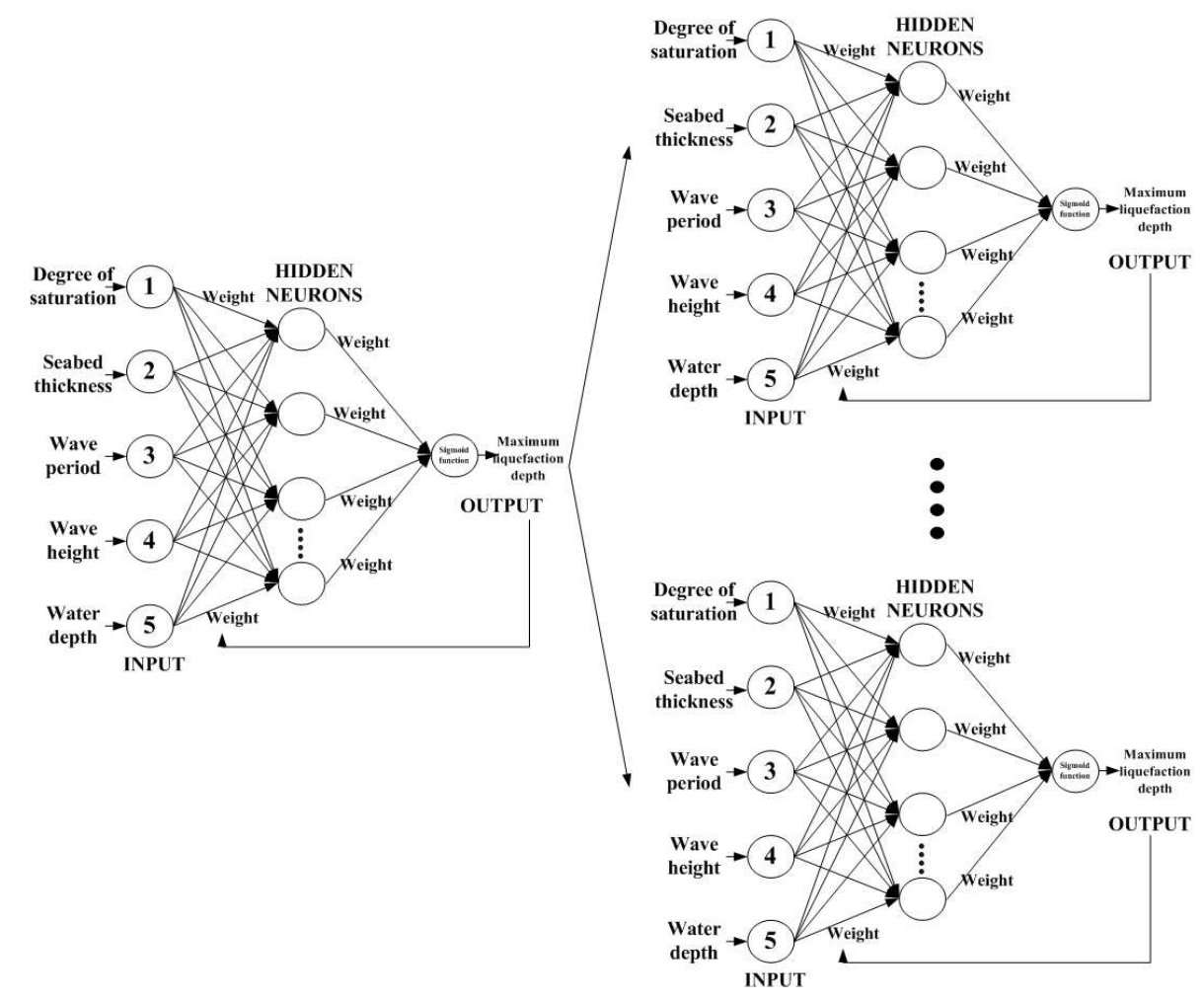

Figure 8: Architecture of MANN model

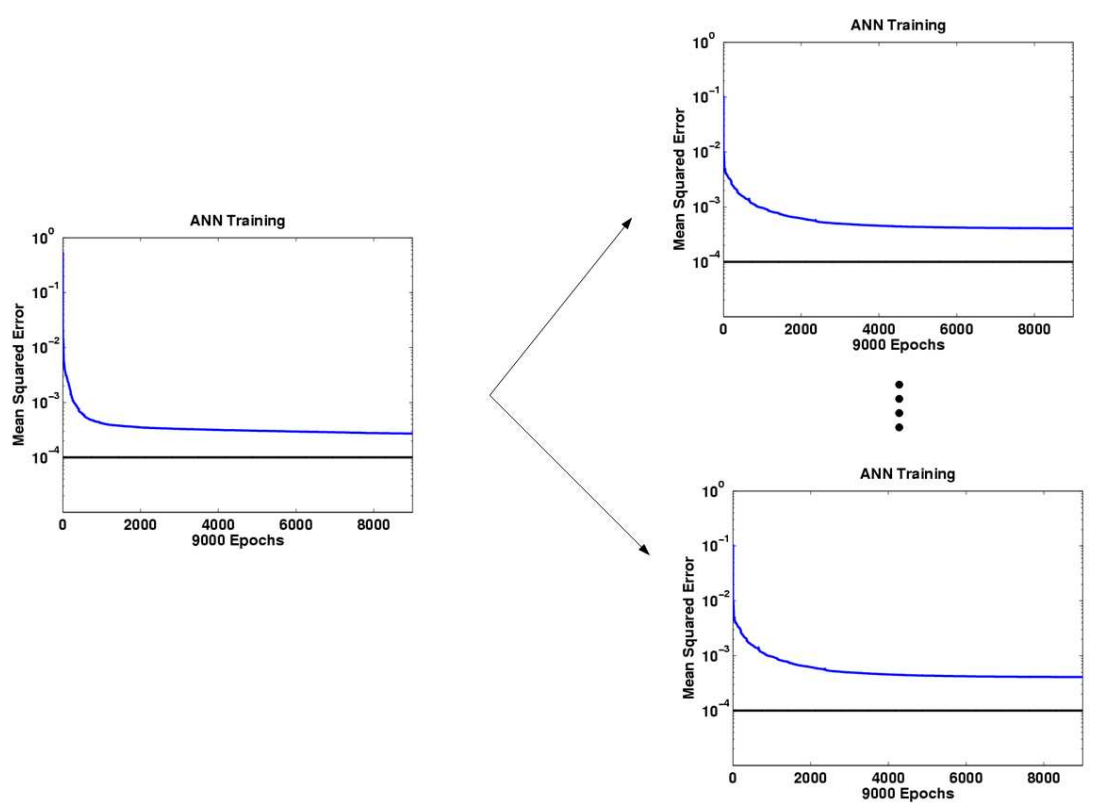

Figure 9: Convergence of MANN model training data 


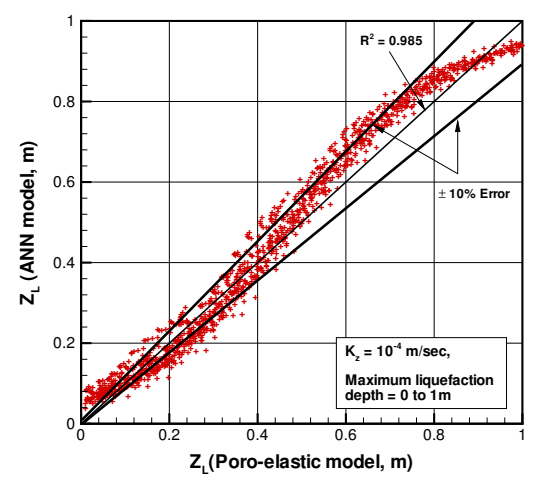

(a) Maximum liquefaction depth between 0 to $1 \mathrm{~m}$

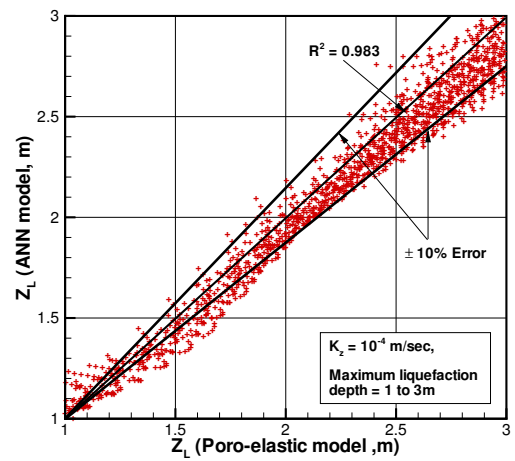

(b) Maximum liquefaction depth between 1 to $3 \mathrm{~m}$

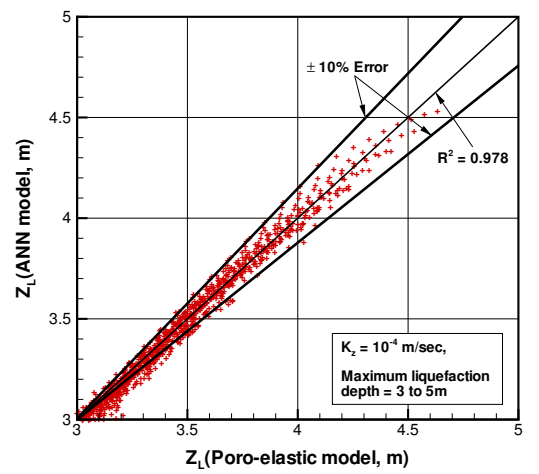

(c) Maximum liquefaction depth between 3 to $5 \mathrm{~m}$.

Figure 10: Comparison of the wave-induced maximum liquefaction depth by the MANN model versus poro-elastic model $\left(K_{z}=10^{-4} \mathrm{~m} / \mathrm{sec}\right)$ 


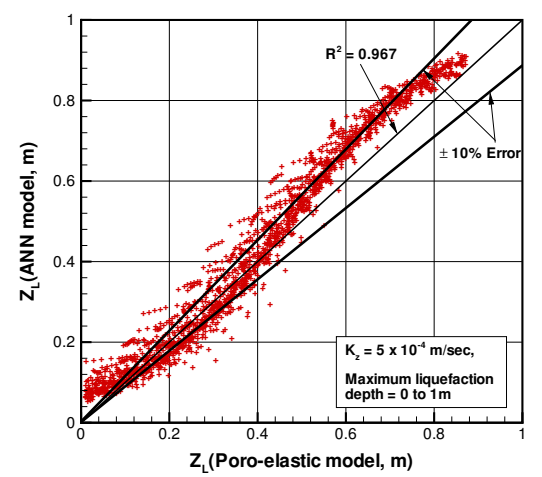

(a) Maximum liquefaction depth between 0 to $1 \mathrm{~m}$

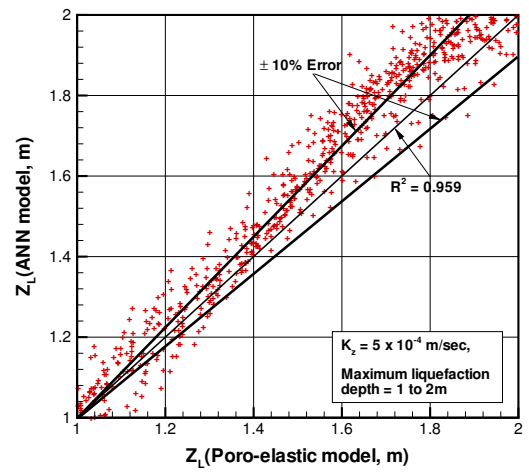

(b) Maximum liquefaction depth between 1 to $2 \mathrm{~m}$

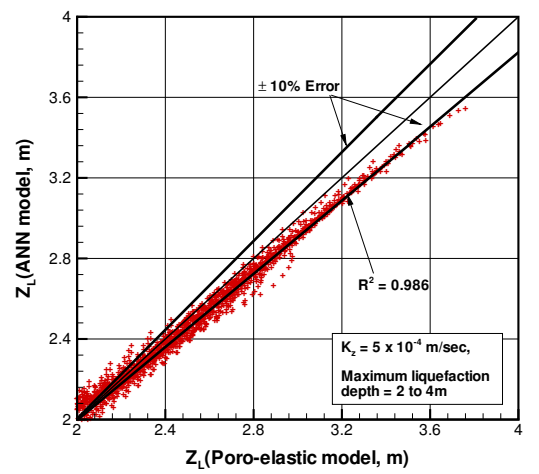

(c) Maximum liquefaction depth between 2 to $4 \mathrm{~m}$.

Figure 11: Comparison of the wave-induced maximum liquefaction depth by the MANN model versus poro-elastic model $\left(K_{z}=5 \times 10^{-4} \mathrm{~m} / \mathrm{sec}\right)$ 